\title{
Safety of Duplex Ultrasound-Assisted Transulnar versus Transradial Arterial Access for Invasive Coronary Procedures: A Search for Safe Alternative Access
}

\author{
Kareem Mahmoud, Ahmed Elamragy, Wael ElNaggar, Mohamed Abou-Elezz \\ Department of Cardiovascular Medicine, Cairo University, Cairo, Egypt \\ ORCID: \\ Kareem Mahmoud: 0000-0002-2398-0000 \\ Ahmed Elamragy: 0000-0001-7344-1825 \\ Mohamed Abou-Elezz: 0000-0002-5399-2563
}

\section{Abstract}

Background: Transulnar approach (TUA) was comparable to the transradial approach (TRA) in terms of efficacy and safety in the presence of extensive operator experience in the TUA. Without adequate experience, there was an increase in time, and number of punctures, to successful access. Objectives: The aim of the study was to compare the in-hospital safety of duplex ultrasound (DUS) assisted TUA to those of DUS-assisted TRA. Methods: All patients had preprocedural DUS assessment. Patients who needed urgent procedures (e.g., ST-elevation myocardial infarction) or had a history of upper extremity arterial disease were excluded. The patients were randomized into TUA or TRA arms. The primary endpoint was a composite of in-hospital vascular access complications (bleeding, limb ischemia, local pain, spasm, and need for crossover). The timing to successful puncture, total number of punctures, total procedural time, fluoroscopy time, amount of contrast, and radiation dose were compared between the two arms as well. Results: A total of 104 patients were randomized into TUA $(n=50)$ or TRA $(n=54)$ arms. There was no statistically significant difference between both arms in terms of the primary endpoint (TUA vs. TRA: $37.5 \%$ vs. $28.6 \%$ respectively, $P=0.33)$. Apart from an increase in the incidence of access site pain $(28 \%$ vs. $7 \%$, respectively, $P=0.006)$, there was no statistically significant difference between the two approaches in terms of time to successful access, number of punctures, total procedural time, fluoroscopy time, or contrast and radiation doses. There was no clinically detectable limb ischemia in both arms on 30-day follow-up. Conclusions: This study demonstrated that there was no difference in safety between DUS-assisted TRA and TUA. In the presence of DUS, TUA becomes a viable alternative arterial access even with the lack of previous TUA experience. More prolonged follow-up is needed to exclude late subclinical events related to the TUA.

Keywords: Duplex ultrasound, radial access, ulnar access, vascular access complications

\section{INTRODUCTION}

The transfemoral approach has several disadvantages, ranging from access site bleeds up to more alarming complications such as retroperitoneal hematoma, arteriovenous fistula, and pseudoaneurysm. These complications translated into increased morbidity, mortality, need for surgery, and blood transfusions and consequently longer hospital stays, higher costs, and worse quality of life..$^{[1,2]}$

\section{Received: 18-06-2020 Revised: 05-08-2020 Accepted: 18-08-2020}

Published Online: 27-11-2020

\begin{tabular}{|l|l|}
\hline \multicolumn{3}{c|}{ Access this article online } \\
\hline Quick Response Code: & Website: \\
& \\
&
\end{tabular}

The transradial approach (TRA) has been proven to have a lower incidence of all these complications in addition to achieving a mortality benefit. This benefit was driven by a lower incidence of bleeding, especially in patients with acute coronary syndrome (ACS) ${ }^{[3,4]}$ The European 2015

Address for correspondence: Dr. Kareem Mahmoud, Department of Cardiovascular, Cairo University, Cairo, Egypt. E-mail: dr.kareem215@yahoo.com

This is an open access journal, and articles are distributed under the terms of the Creative Commons Attribution-NonCommercial-ShareAlike 4.0 License, which allows others to remix, tweak, and build upon the work non-commercially, as long as appropriate credit is given and the new creations are licensed under the identical terms.

For reprints contact: WKHLRPMedknow_reprints@wolterskluwer.com

How to cite this article: Mahmoud K, Elamragy A, ElNaggar W, Abou-Elezz M. Safety of duplex ultrasound-assisted transulnar versus transradial arterial access for invasive coronary procedures: A search for safe alternative access. Int J Cardiovasc Acad 2020;6:150-6. 
non-ST-segment elevation myocardial infarction Guidelines, the 2017 ST-elevation myocardial infarction (STEMI) Guidelines and the 2018 Revascularization Guidelines all label the TRA as the preferred access to reduce percutaneous coronary intervention (PCI)-related bleeding risk in these settings, provided that operator skills meet the required standards. ${ }^{[5-7]}$ The American Heart Association followed suit in 2018, releasing a scientific statement recommending a radial-first approach in all patients, with a graduated level of center and operator experience condition before TRA is pursued in patients with ACS. ${ }^{[8]}$

An alternative access may be needed in some situations. For example, there could be a need to preserve their arteries for other procedures such as hemodialysis or coronary artery bypass grafting (CABG). In other times, radial access could not be established (e.g. refractory spasm and marked tortuosity). In most such situations, the femoral artery is used. The other forearm artery, the ulnar artery, could be an alternative vascular access to the radial artery in some of these cases. However, there are several anatomical considerations that should be kept in mind. The ulnar artery is deeper, has no bony landmark for compression, less in-line with brachial artery and is in close relation to ulnar nerve. ${ }^{[9]}$

Duplex ultrasound (DUS) guidance has already been used to improve rates of successful cannulation and reduce complications in both transradial ${ }^{[10]}$ and transfemoral ${ }^{[11]}$ approaches. Theoretically then, DUS could be the tool needed to overcome the procedural hurdles associated with transulnar approach (TUA) and make it a viable option even when the operator lacks previous extensive transulnar experience.

Our study aimed to compare the intraprocedural and short-term postprocedural safety of the DUS assisted TUA to the TRA in the setting of invasive coronary angiography.

\section{Methods}

This was a prospective, randomized, single-center trial. We used consecutive sampling from April 2018 to December 2018 to sample participants. Patients who were scheduled for invasive coronary procedures at our center on our operators' workdays were screened for eligibility of inclusion in the study. All patients were scheduled for right forearm access except when otherwise deemed by the clinical scenario, for example, CABG patients with left internal mammary artery grafts, or right upper extremity disease precluding arterial access from that arm. Written informed consent was obtained from all subjects enrolled in the study. The study was approved by the local ethics committee.

\section{Exclusion criteria}

- STEMI or very high risk NSTEACS

- Previous or scheduled procedures involving the ulnar or radial arteries, for example, arteriovenous fistulae and radial artery graft for $\mathrm{CABG}$

- $\quad$ Severe upper extremity arterial disease (rest pain, fixed color changes, or tissue loss)
- Complex PCI needing femoral access

- Small ipsilateral radial or ulnar arterial diameter $(<2 \mathrm{~mm})$ or occlusion of planned access vessel by DUS.

\section{Clinical data}

The following history was obtained from each patient: Age, sex, diabetes mellitus, hypertension, renal impairment (serum creatinine $>1.5 \mathrm{mg} / \mathrm{dL}$ ), and upper extremity arterial disease or vascular procedures. Bilateral radial and ulnar pulsations were examined and noted. Allen test was not done in this study as, in previous studies, it did not correlate with the adequacy of the hand circulation. ${ }^{[12]}$

\section{Duplex ultrasound}

Preprocedural DUS using a Philips EPIQ 7 device with an eL18-4 linear probe, noting the ulnar and radial vessel diameter, patency, peak systolic velocity and any anatomical abnormalities. Using a permanent marker with a bullet tip, $3 \mathrm{~mm}$ diameter marks were then added, one above and one below the ultrasound probe position along the course of each forearm artery, with the distal mark placed at the level of the proximal wrist crease. Figure 1 shows an example of assessment of the ulnar artery by DUS.

\section{Patient randomization}

We used the Google random number generator to randomize patients into TUA and TRA arms. It was set to a minimum of 1 and a maximum of 100 . Each patient then got a number: Odd numbers were allocated into the TUA arm, and even numbers into the TRA arm.

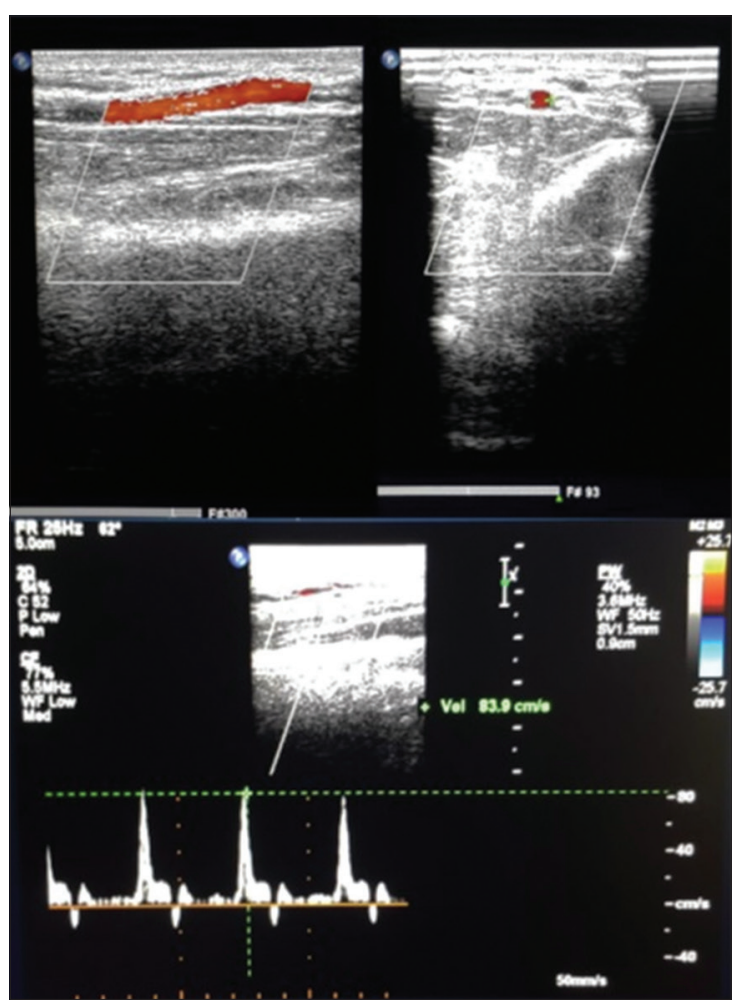

Figure 1: Assessment of the ulnar artery by duplex ultrasound; Top left: along its axis; Top right: cross sectional; Bottom: assessment of peak systolic velocity 


\section{Patient preparation}

Intravenous (IV) access was achieved away from the patient's planned access forearm. Adequate disinfection of the forearm was done with liquid povidone-iodine. Five minutes before the attempt, $3 \mathrm{mg}$ bolus IV midazolam was administered, aiming for mild sedation.

\section{Operators}

The procedures were done by two interventional cardiologists with over 150 procedures involving forearm arterial access per year (the majority TRA, with few TUA procedures). The operator decides the desired exact puncture site, using the DUS marks and the pulsations as a guide.

\section{Access details}

Local anesthesia was achieved with subcutaneous injection of $2 \mathrm{ml}$ lidocaine at the puncture site. The sheath used was a Merit Medical $^{\mathrm{TM}}$ Transradial Prelude PSI Version C 21G, 6F, $11 \mathrm{~cm}$ sheath with a $4 \mathrm{~cm}$ needle. After sheath insertion, a cocktail of nitroglycerin $100 \mu \gamma$, verapamil $2.5 \mathrm{mg}$ and unfractionated heparin 5000-10000 U were given intra-arterially.

\section{Data collected in the cath-lab}

Timing of the procedure starts with the first skin puncture. The number of attempts, timing to puncture (in seconds), local access complications (pain, spasm, and hematoma) were noted. Decision of cross-over and vessel to cross-over to was left to the discretion of the operator. The alternative access and reason for crossing over were noted. The presence of loops or marked tortuosity during guide-wire insertion was recorded. Tortuosity and loops were navigated through one or a combination of the following: catheter manipulation, catheter-assisted tracking techniques, and/or deep inspiration. Procedural obstacles, such as difficulty in engaging the coronaries, were noted.

At the end of the procedure, the type of procedure itself (diagnostic coronary angiography or PCI), number of stents, the total procedure time (in minutes), contrast volume used, radiation dose and fluoroscopy time were noted.

\section{Postprocedural details}

After the procedure, the sheath was removed immediately after the end of the procedure, ensuring hemostasis. In case of difficult sheath removal due to arterial spasm, a warm foment doused in nitroglycerin was placed over the skin covering the sheath track, followed by gentle withdrawal of the sheath. A Terumo TR-band ${ }^{\circledR}$ compression device was used to seal the puncture site and was closely monitored. It was removed $2-4 \mathrm{~h}$ after the procedure. During their time in the recovery room, the patient's vitals were noted, and local complications such as pain, hematoma, bleeding, early ischemia and local mononeuropathy, for example, sensory loss were recorded clinically. Bleeding was classified into trivial, mild, moderate, severe, and life-threatening bleeding according to the 2017 ESC focused update on dual antiplatelet therapy in coronary artery disease: ${ }^{[13]}$

- Trivial: Any bleeding not requiring intervention or further evaluation
- Mild: Bleeding that requires intervention without further hospitalization

- Moderate: Bleeding associated with significant blood loss $(>3-5 \mathrm{~g} / \mathrm{dL})$ and/or requiring hospitalization, which is hemodynamically stable and not rapidly evolving

- Severe: Bleeding associated with significant blood loss $(>5 \mathrm{~g} / \mathrm{dL})$ requiring hospitalization, which is hemodynamically stable and not rapidly evolving

- Life threatening: Any severe active bleeding that puts the patient's life immediately at risk, for example, hemodynamic instability.

Arterial pulsations after compression band removal were noted. We discharged patients once they were deemed fit by the operator.

\section{Primary endpoints}

In-hospital vascular access complications (composite of bleeding, limb ischemia, local pain, spasm, and need for crossover).

\section{Secondary endpoints}

- In-hospital access complications access individual points (local pain, spasm, need for crossover, bleeding, or limb ischemia)

- One-month clinical follow-up for limb ischemia

- Procedural details: timing to successful puncture, number of attempts, total procedural time, fluoroscopy time, radiation dose, and contrast volume.

\section{Statistical analysis}

Continuous data are displayed as mean \pm standard deviation or median and interquartile range, while categorical data are displayed as number and percentage. Comparison between continuous data was done using Student's unpaired $t$-test, while comparison between categorical data was done using a Chi-square test. Findings with a two-tailed $P<0.05$ were considered statistically significant. Analysis was done in the Statistical Package for Social Sciences program (SPSS) version 24.0. Armonk, NY, USA: IBM Corp.

\section{RESULTS}

We screened 140 patients presenting for invasive coronary angiography. We excluded 36 patients mostly due to emergency PCI. We randomized each of the remaining 104 patients into one of two groups: the TRA group (54 patients) and the TUA group (50 patients) as seen in Figure 2.

The baseline characteristics of the included patients in each arm are presented in Table 1. There was no statistically significant difference between both arms regarding age, gender, or risk factors. In the both groups, about three quarters of the patients included presented with stable ischemic heart disease with either positive noninvasive stress tests or chest pain for differential diagnosis. On the other hand, only $19 \%$ of the patients included presented with an ACS. Seven percent of the patients in each group were undergoing catheterization as 


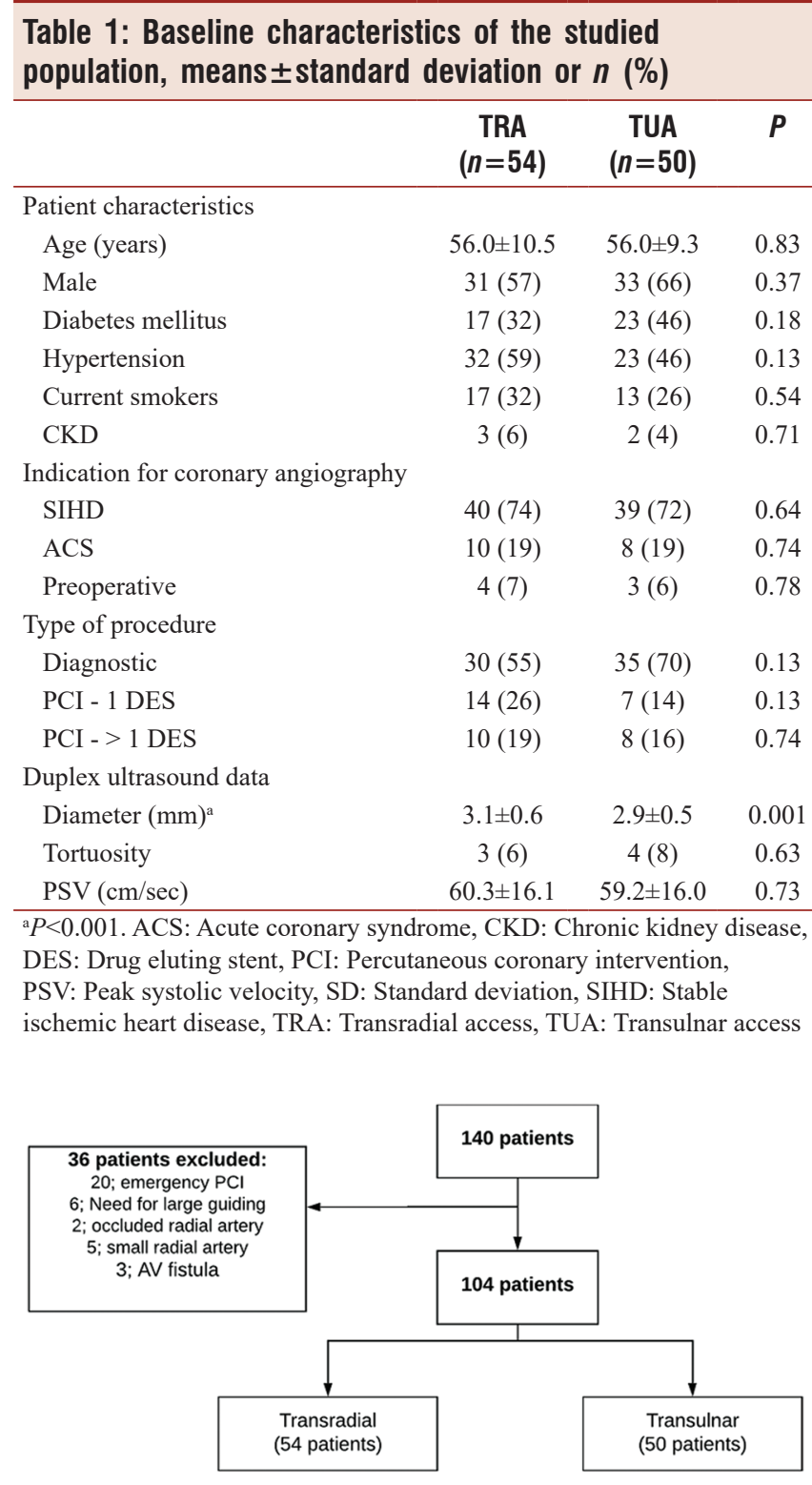

Figure 2: Flowchart of the studied patients

part of their cardiothoracic preoperative preparation (all were scheduled for valve surgeries).

There was no significant difference between both arms regarding DUS data (except for larger diameter of radial artery) as shown in Table 1.

Procedural data are summarized in Table 2. There was no significant difference between both groups regarding the composite endpoints of limb ischemia, bleeding, procedural pain, spasm, and need to cross-over to another access. The incidence of intraprocedural and postprocedural pain was higher in the translunar group. However, all these patients were free of pain on discharge. There was no significant difference between both arms regarding the incidence of spasm or rate of cross-over. Of the 8 cross-overs in the TRA group, 2 were to the TUA and 6 were to the transfemoral approach. Out of the
Table 2: Procedural data in each arm, means \pm standard deviation (or median and interquartile range) or $\boldsymbol{n}(\%)$

\begin{tabular}{|c|c|c|c|}
\hline & $\operatorname{TRA}(n=54)$ & TUA $(n=50)$ & $P$ \\
\hline Primary endpoint ${ }^{\mathrm{a}}$ & $16(28.6 \%)$ & $18(37.5 \%)$ & 0.33 \\
\hline \multicolumn{4}{|l|}{ Access adverse events } \\
\hline Pain $^{2}$ & $4(7)$ & $14(28)$ & 0.006 \\
\hline Spasm & $6(11)$ & $3(6)$ & 0.96 \\
\hline Crossovers & $8(15)$ & $8(16)$ & 0.87 \\
\hline Hematoma $<1 \mathrm{~cm}$ & $2(4)$ & $1(2)$ & 0.60 \\
\hline Hand ischemia & 0 & 0 & NA \\
\hline \multicolumn{4}{|l|}{ Post-procedural complications } \\
\hline Persistent access site pain ${ }^{\mathrm{b}}$ & $0(0)$ & $7(14)$ & 0.004 \\
\hline Difficult sheath removal & $1(2)$ & $1(2)$ & 0.96 \\
\hline $1 \mathrm{~m}$ clinical limb ischemia & $0(0)$ & $0(0)$ & NA \\
\hline \multicolumn{4}{|l|}{ Number of punctures ${ }^{c}$} \\
\hline Median & 2 & 2 & 0.20 \\
\hline IQR & $1-2.25$ & $1-3$ & \\
\hline \multicolumn{4}{|l|}{ Time to access $(\mathrm{sec})^{\mathrm{c}}$} \\
\hline Median & 69 & 79 & 0.86 \\
\hline IQR & $30.8-154.5$ & $44.8-180.3$ & \\
\hline \multicolumn{4}{|l|}{ Total procedure time (min) } \\
\hline Total & $34.4 \pm 22.1$ & $30.4 \pm 24.1$ & 0.38 \\
\hline Diagnostic & $20.0 \pm 11.4$ & $17.0 \pm 5.4$ & 0.19 \\
\hline PCI - 1 DES & $42.9 \pm 14.0$ & $38.8 \pm 6.5$ & 0.37 \\
\hline PCI - > 1DES & $64.2 \pm 19.2$ & $77.8 \pm 15.8$ & 0.12 \\
\hline \multicolumn{4}{|l|}{ Fluoroscopy time (min) } \\
\hline Total & $13.3 \pm 11.0$ & $11.4 \pm 14.7$ & 0.47 \\
\hline Diagnostic & $7.3 \pm 6.2$ & $4.9 \pm 3.1$ & 0.07 \\
\hline PCI - 1 DES & $15.9 \pm 9.2$ & $13.1 \pm 4.4$ & 0.36 \\
\hline PCI - > 1DES & $27.1 \pm 10.4$ & $36.6 \pm 21.4$ & 0.27 \\
\hline \multicolumn{4}{|l|}{ Total radiation $(\mathrm{Gy})$} \\
\hline Total & $2.2 \pm 1.6$ & $2.0 \pm 1.7$ & 0.71 \\
\hline Diagnostic & $1.2 \pm 0.8$ & $0.9 \pm 0.3$ & 0.05 \\
\hline PCI - 1 DES & $2.6 \pm 1.0$ & $2.2 \pm 0.5$ & 0.18 \\
\hline PCI - > 1DES & $4.4 \pm 0.1$ & $4.4 \pm 0.5$ & 0.56 \\
\hline \multicolumn{4}{|l|}{ Total contrast volume $(\mathrm{ml})$} \\
\hline Total & $110.4 \pm 73.1$ & $91.1 \pm 71.4$ & 0.19 \\
\hline Diagnostic & $64.7 \pm 29.5$ & $52.9 \pm 11.8$ & 0.05 \\
\hline PCI - 1 DES & $135.7 \pm 45.7$ & $114.3 \pm 24.4$ & 0.27 \\
\hline PCI - > 1DES & $207.5 \pm 83.4$ & $228.1 \pm 64.7$ & 0.28 \\
\hline
\end{tabular}

${ }^{a}$ Composite of limb ischemia, bleeding, access site pain, spam and need for crossover, ${ }^{b} P<0.01$, ${ }^{\mathrm{c}}$ Data are represented as median and IQR. DES: Drug eluting stent, IQR: Interquartile range, PCI: Percutaneous coronary intervention, SD: Standard deviation, TRA: Transradial access, TUA: Transulnar access

8 cross-overs in the TUA group, 4 were to the TRA and 4 were to the transfemoral approach. Failure of arterial cannulation was the most common reason for cross-over (11 patients). Other causes of cross-over were intolerable pain or spasm, marked tortuosity and inadequate guiding support.

There was no significant difference between both the groups regarding the all procedural details as demonstrated in Table 2 .

All PCI procedures included were successful, with resultant TIMI III flow at the end of the procedure. There were no deaths, 
major adverse cardiovascular events (MACE) or hemodynamic instability events during any of the procedures.

Monitoring patients until discharge on the same day or the next day, postprocedural complications, again, were detected in a limited number of patients. Out of the 14 patients who had intraprocedural access site pain in the TUA, 7 patients had persistent pain after the procedure. No patients in the TRA reported such carried-over pain. In all these 7 patients, none of them had persistent pain after compression band removal.

One patient in each arm had severe spasm precluding sheath removal $(P=0.956)$ that was managed as described in methods section. Trivial bleeding, in the form of a small access site hematoma $\leq 1 \mathrm{~cm}$ in diameter, was present in 3 patients in the study, 2 patients in the TRA and 1 patient in the TUA. The hematomas were not associated with any peripheral vascular complications. None of the patients had significant bleeding, peripheral ischemia or local mononeuropathy. There were no reported cases of death of myocardial infarction in the study. There was no clinical evidence of hand or forearm ischemia in both groups on 1 month clinical follow-up.

\section{Discussion}

In this randomized, single-center trial, we used DUS as a guide to TRA and TUA in invasive coronary procedures. We found no significant difference between both approaches in terms of in-hospital composite events of limb ischemia, bleeding, pain, spasm, and need for crossover. Furthermore, there was no difference in number of punctures, access time and all other procedural details between the two arms. The fact that these results were seen regardless of operator experience contrasted with the AJULAR study: The AJULAR study had previously recommended that a minimum experience prerequisite of 100 previous TUA cases is needed before obtaining similar results from the TUA arm. ${ }^{[14]}$

Access site pain was statistically higher in the TUA despite using the same local anesthesia approach. This could be due to its proximity to the palmar cutaneous branch of the ulnar nerve, especially its distal part. Needle trauma to this branch or to the ulnar nerve itself causes shooting pain along their sensory distribution. The incidence of spasm and crossover rates were similar between both arms; so were other procedural aspects including the total procedural duration, fluoroscopy time, contrast volume, radiation exposure, and procedure outcome.

Short-term postprocedural complications were also similar between both arms; in terms of clinical absence of pulsations or other manifestations of distal peripheral vascular disease, bleeding, or injury to the local nerves. The absence of major cardiovascular complications (e.g. death and myocardial infarction), moderate or severe bleeding or peripheral vascular events during hospital stay in our study underlines the advantages of the forearm access in general. There were no clinically detectable ischemic events of the forearm and hand related to the accessed artery on 1 month follow-up.
Clinically evident manifestations of upper extremity arterial disease (by history or examination) were among the exclusion criteria. Maniotis et al. ${ }^{[12]}$ determined that preprocedural Allen's test should not be routinely performed before TRA in invasive coronary angiography, as there was no difference in efficacy and safety of the access whether Allen's test was positive or not. As a result, we did not perform preprocedural Allen test. Cross-over to the ipsilateral forearm artery in case of access failure (even if the artery was punctured without subsequent cannulation) was done without complications in 6 patients. The absence of peripheral vascular complications in our study suggests that abandoning Allen's test is safe with no unfavorable consequences after crossing over to TUA due to failed TRA. Kedev et al. ${ }^{[15]}$ supported the safety of the TUA after a failed TRA. Similar results were also produced by the multicenter SWITCH registry. ${ }^{[16]}$

The first large scale study comparing TUA and TRA was the PCVI-CUBA study. ${ }^{[17]}$ This study concluded that the two accesses had a similar safety and efficacy profile, however the mean number of punctures was greater in the TUA.

The larger AURA of ARTEMIS trial ${ }^{[18]}$ (903 patients) was terminated early in 2013 due to significant increase in the number of crossovers, the number of punctures, access time, fluoroscopy time, contrast volume, procedure time, the incidence of vessel occlusion at 60 days and MACE at 60 days in the TUA; findings that are discordant with our study. However, the participating operators lacked adequate transulnar experience and the patients were enrolled regardless of the quality of the ulnar artery pulse quality. These 2 points were further reinforced by 2 more trials: Liu et al. ${ }^{[19]}$ and the AJULAR study. ${ }^{[14]}$ In both studies, the operators had extensive TUA experience. Their results were more in line with our study as well as PCVI-CUBA trial. The AJULAR study concluded that a learning curve of at least 100 patients must first be achieved in order to reproduce their favorable results. Our study suggested that operators without this experience can still go transulnar with DUS assistance, with an efficacy and safety profile comparable to TRA.

The PCVI-CUBA study ${ }^{[17]}$ reported transient lightening flash pain on the ulnar side of the hand in 3 out of 216 patients. In our study, TUA had significantly higher acute pain (28\% vs. $7 \%$, $P=0.006$ ). However, the incidence of spasm was similar between both approaches. This was concordant with AJULAR study, ${ }^{[14]}$ but discordant with the AURA of ARTEMIS study ${ }^{[18]}$ in which the absolute number of spasms were greater in the TUA. In both studies, the differences did not reach statistical significance. However, studies by Liu et al. ${ }^{[19]}$ and the AJULAR in elderly ${ }^{[20]}$ agree that the TUA was associated with significantly less incidence of spasm. This can be explained anatomically by the fewer alpha-adrenergic receptors on the ulnar artery relative to the radial one.

The similarity in access failures between the two approaches in our study falls in line with the AJULAR, Liu et al., and PCVI-CUBA studies. The increased incidence of access site 
pain in the TUA in our study did not translate to a difference in crossover rate; one patient only from each arm was subjected to an access site crossover due to intractable pain or spasm. This is probably because the pain was only transient in half of the cases, and tolerable in most of the other half.

The procedural and postprocedural results of our study were in line with previous studies. ${ }^{[14,17,19]}$ Liu et al. ${ }^{[19]}$ reported a significantly lower incidence of forearm hematoma in the TUA. This complication was not encountered in our study, probably due to a smaller sample size.

Previous studies comparing DUS data of the radial and ulnar arteries were conflicting. ${ }^{[15,17,21-23]}$ Our findings were consistent with the findings of Baumann and Roberts, ${ }^{[21]}$ Kim et al. ${ }^{[22]}$ and Huzjan et al..$^{[23]}$ all reported a larger average diameter of the radial artery and no statistically significant difference in peak systolic velocity.

The results of our study place an elephant in the room; a question that must be addressed eventually: Since the TUA is as closely-matched to the TRA as ever, does the former bring enough to the table to replace the latter as the default strategy rather than being just an alternative? At present, while the TUA showed no major difference from TRA especially with the guidance of DUS, it does not appear to provide a clear advantage and its perceived edge over the TRA appears limited to very specific scenarios.

This was not the target of the study though. The true aim was to break the ice between the average coronary interventional cardiologist and the TUA, highlighting it as an alternative to the standard TRA when the scenario arises, ousting the transfemoral access and sparing the patients from its potential risks.

The TUA still does not overcome all the drawbacks of the TRA. There are still settings where the application of any forearm access is not suitable, for example, forearm arteriovenous fistulae for dialysis or challenging tortuosities above the level of the forearm arteries. In these settings, the TUA does not provide any benefit over the TRA. Moreover, the TUA will not offer much to operators who are not already trained in the TRA.

\section{Study limitations}

Investigator bias was a potential source of error. This was minimized using independent observers to acknowledge events during timing and examine for complications. Patients with STEMI were excluded. Thus, the results do not reflect the practice in emergency situations. Long-term follow-up by clinical evaluation and DUS is needed to detect late subclinical events.

\section{Conclusions}

Our study showed that with the use of DUS, there was no significant difference in safety between the transulnar and the TRA in the setting of invasive coronary angiography, even when previous TUA experience is lacking. In the presence of DUS, the TUA becomes a viable alternative to the TRA in many settings where the latter is not feasible. A study on a larger scale with more prolonged follow-up could further build up on these findings.

\section{Financial support and sponsorship \\ Nil.}

\section{Conflicts of interest}

There are no conflicts of interest.

\section{References}

1. Yatskar L, Selzer F, Feit F, Cohen HA, Jacobs AK, Williams DO, et al. Access site hematoma requiring blood transfusion predicts mortality in patients undergoing percutaneous coronary intervention: Data from the National Heart, Lung, and Blood Institute Dynamic Registry. Catheter Cardiovasc Interv 2007;69:961-6.

2. Ndrepepa G, Berger PB, Mehilli J, Seyfarth M, Neumann FJ, Schömig A, et al. Periprocedural bleeding and 1-year outcome after percutaneous coronary interventions: Appropriateness of including bleeding as a component of a quadruple end point. J Am Coll Cardiol 2008;51:690-7 .

3. Jolly SS, Yusuf S, Cairns J, Niemelä K, Xavier D, Widimsky P, et al. Radial versus femoral access for coronary angiography and intervention in patients with acute coronary syndromes (RIVAL): A randomised, parallel group, multicentre trial. Lancet 2011;377:1409-20.

4. Valgimigli M, Gagnor A, Calabró P, Frigoli E, Leonardi S, Zaro T, et al. Radial versus femoral access in patients with acute coronary syndromes undergoing invasive management: A randomised multicentre trial. Lancet 2015;385:2465-76.

5. Roffi M, Patrono C, Collet JP, Mueller C, Valgimigli M, Andreotti F, et al. 2015 ESC Guidelines for the management of acute coronary syndromes in patients presenting without persistent ST-segment elevation: Task Force for the Management of Acute Coronary Syndromes in Patients Presenting without Persistent ST-Segment Elevation of . Eur Heart J 2016;37:267-315.

6. Ibanez B, James S, Agewall S, Antunes MJ, Bucciarelli-Ducci C, Bueno H, et al. 2017 ESC Guidelines for the management of acute myocardial infarction in patients presenting with ST-segment elevation: The task force for the management of acute myocardial infarction in patients presenting with ST-segment elevation of the European Socie. Eur Heart J 2018;39:119-77.

7. Neumann FJ, Sousa-Uva M, Ahlsson A, Alfonso F, Banning AP, Benedetto U, et al. 2018 ESC/EACTS Guidelines on myocardial revascularization. Eur Heart J 2019;40:87-165.

8. Mason PJ, Shah B, Tamis-Holland JE, Bittl JA, Cohen MG, Safirstein J, et al. An update on radial artery access and best practices for transradial coronary angiography and intervention in acute coronary syndrome: A scientific statement from the American Heart Association. Circ Cardiovasc Interv 2018;11:e000035.

9. Kar S. Transulnar cardiac catheterization and percutaneous coronary intervention: Techniques, transradial comparisons, anatomical considerations, and comprehensive literature review. Catheter Cardiovasc Interv 2017;90:1126-34.

10. Seto AH, Roberts JS, Abu-Fadel MS, Czak SJ, Latif F, Jain SP, et al. Real-time ultrasound guidance facilitates transradial access: RAUST (Radial Artery access with Ultrasound Trial). JACC Cardiovasc Interv 2015;8:283-91.

11. Seto AH, Abu-Fadel MS, Sparling JM, Zacharias SJ, Daly TS, Harrison AT, et al. Real-time ultrasound guidance facilitates femoral arterial access and reduces vascular complications: FAUST (Femoral Arterial Access With Ultrasound Trial). JACC Cardiovasc Interv 2010;3:751-8.

12. Maniotis C, Koutouzis M, Andreou C, Lazaris E, Tsiafoutis I, Zografos T, et al. Transradial Approach for Cardiac Catheterization in Patients With Negative Allen's Test. J Invasive Cardiol 2015;27:416-20.

13. Valgimigli M, Bueno H, Byrne RA, Collet JP, Costa F, Jeppsson A, et al. 2017 ESC focused update on dual antiplatelet therapy in coronary artery 
disease developed in collaboration with EACTS: The Task Force for dual antiplatelet therapy in coronary artery disease of the European Society of Cardiology (ESC) and of the European . Eur Heart J 2018;39(3):21360.

14. Gokhroo R, Kishor K, Ranwa B, Bisht D, Gupta S, Padmanabhan D, et al. Ulnar artery interventions non-inferior to radial approach: AJmer Ulnar ARtery (AJULAR) Intervention Working Group Study Results. J Invasive Cardiol 2016;28:1-8.

15. Kedev S, Zafirovska B, Dharma S, Petkoska D. Safety and feasibility of transulnar catheterization when ipsilateral radial access is not available. Catheter Cardiovasc Interv 2014;83:E51-60.

16. Agostoni P, Zuffi A, Faurie B, Tosi P, Samim M, Belkacemi A, et al. Same wrist intervention via the cubital (ulnar) artery in case of radial puncture failure for percutaneous cardiac catheterization or intervention: The multicenter SWITCH registry. Int J Cardiol 2013;169:52-6.

17. Aptecar E, Pernes JM, Chabane-Chaouch M, Bussy N, Catarino G, Shahmir A, et al. Transulnar versus transradial artery approach for coronary angioplasty: The PCVI-CUBA study. Catheter Cardiovasc Interv 2006;67:711-20.

18. Hahalis G, Tsigkas G, Xanthopoulou I, Deftereos S, Ziakas A, Raisakis $\mathrm{K}$, et al. Transulnar compared with transradial artery approach as a default strategy for coronary procedures: A randomized trial. The Transulnar or Transradial Instead of Coronary Transfemoral
Angiographies Study (the AURA of ARTEMIS Study). Circ Cardiovasc Interv 2013;6:252-61.

19. Liu J, Fu XH, Xue L, Wu WL, Gu XS, Li SQ. A comparative study of transulnar and transradial artery access for percutaneous coronary intervention in patients with acute coronary syndrome. J Interv Cardiol 2014;27:525-30

20. Chandra K, Gokhroo R. Ajmer ulnar artery access in elderly (AJULAR in elderly): A single center prospective randomized control study. J Am Coll Cardiol 2018;71:A1268.

21. Baumann F, Roberts JS. Real time intraprocedural ultrasound measurements of the radial and ulnar arteries in 565 consecutive patients undergoing cardiac catheterization and/or percutaneous coronary intervention via the wrist: Understanding anatomy and anomalies may improve access success. J Interv Cardiol 2015;28:574-82.

22. Kim SY, Lee JS, Kim WO, Sun JM, Kwon MK, Kil HK. Evaluation of radial and ulnar blood flow after radial artery cannulation with 20- and 22-gauge cannulae using duplex Doppler ultrasound. Anaesthesia 2012;67:1138-45.

23. Huzjan R, Brkljacić B, Delić-Brkljacić D, Biocina B, Sutlić Z. B-mode and color Doppler ultrasound of the forearm arteries in the preoperative screening prior to coronary artery bypass grafting. Coll Antropol 2004;28:235-41 